\title{
Réflexions sur les principes juridiques de l'éducation inclusive au Brésil légalité, droit à la différence, équité
}

Reflections on the principles of inclusive education in Brazil: equality, right to difference, equity

Reflexiones sobre los principios de la educación inclusiva en Brasil : igualdad, derecho a la diferencia, equidad

Überlegungen zu den Prinzipien der inklusiven Erziehung in Brasilien:

Gleichberechtigung, Recht auf Anderssein, Billigkeit

\section{Carlos Roberto Jamil Cury}

\section{OpenEdition}

\section{Journals}

Édition électronique

URL : http://journals.openedition.org/rechercheformation/486

DOI : 10.4000/rechercheformation.486

ISSN : 1968-3936

Éditeur

ENS Éditions

\section{Édition imprimée}

Date de publication : 1 juin 2009

Pagination : 41-53

ISBN : 978-2-7342-1164-8

ISSN : 0988-1824

\section{Référence électronique}

Carlos Roberto Jamil Cury, « Réflexions sur les principes juridiques de l'éducation inclusive au Brésil légalité, droit à la différence, équité », Recherche et formation [En ligne], 61 | 2009, mis en ligne le 01 juin 2013, consulté le 02 mai 2019. URL : http://journals.openedition.org/rechercheformation/486 ; DOI : 10.4000/rechercheformation.486 


\section{RÉFLEXIONS SUR LES PRINCIPES JURIDIQUES DE L'ÉDUCATION INCLUSIVE AU BRÉSIL Éqalité, droit à la différence, équité}

\section{Carlos Roberto Jamil CURY*}

Résumé L'article examine comment la loi brésilienne peut à la fois rejeter le principe de toute discrimination et justifier un traitement spécifique pour les enfants "à besoins éducatifs particuliers". Les notions de justice, d'égalité, d'équité sont confrontées au cas des minorités (culturelles, linguistiques, ethniques). De même, le droit universel à l'éducation doit traiter des situations singulières, qui exigent une " pédagogie spéciale", des contenus appropriés ou des procédures alternatives. Les maîtres doivent être formés à ce traitement au cas par cas, éloigné d'une tradition scolaire égalitariste, mais juridiquement bien-fondé.

Mots clés: besoins éducatifs particuliers, juridiction, inclusion, équité.

L'éducation inclusive, comme l'indique l'épithète, fait référence au verbe inclure qui signifie mettre quelque chose/quelqu'un dans un espace. Le verbe latin "in-claudere » signifie « in-clure » dans le sens $d^{\prime}$ " en-fermer ». Le terme cloître indique bien cet espace dont quelques-uns font partie, espace délimité, fermé aux autres. In-clure serait donc faire entrer dans la clôture du cloître, c'est-à-dire faire entrer dans un endroit qui comprend un mode de fonctionnement déterminé : pour y jouir des avantages réservés aux entrants. Mais le même terme peut désigner aussi un espace pire que celui d'où vient le sujet. Exclu de la convivialité sociale, le prisonnier est " inclus » dans les grilles de la prison. Dans le cadre d'une sphère sociale, l'inclusion peut convenir aussi bien à une mobilité ascendante que descendante.

* - Carlos Roberto Jamil Cury, Pontifícia Universidade Católica de Minas Gerais, Belo Horizonte, Brésil 
Il faut garder ce fait en mémoire quand on parle de l'inclusion de façon absolve, abstraite, sans autre précision. En effet, l'inclusion et l'exclusion (de celvi qui est dedans vs dehors) entretiennent une relation dialectique, puisque l'une $n^{\prime}$ existe pas sans l'autre. Exclure est aussi bien l'action de rejeter que celle d'interdire l'entrée. Parler d'éducation inclusive suppose donc de revenir sur les thèmes de l'égalité, de l'inégalité ou de la discrimination et de réfléchir sur ce que désignent ces concepts. Dans son principe, l'éducation inclusive correspond à une modalité de scolarisation où ceux qui étudient ont les mêmes droits que leurs pairs, sans discrimination de sexe, de race, d'ethnie, de religion et de capacité. Ils disposent donc du droit de fréquenter les mêmes établissements et de participer aux activités des élèves de leur âge.

Cependant, cette scolarisation ne peut se faire, pour certains, sans une prise en compte de leurs « besoins particuliers »: il faut des adaptations qui rendent possible leur participation aux activités normalement prévues pour leur classe d'âge. Cette double contrainte (traiter de façon à la fois égalitaire et différenciée) pose de redoutables problèmes éthiques et juridiques. Quels sont les principes qui fondent les politiques $d$ 'inclusion dans les sociétés contemporaines? Comment a été construit le cadre juridique qui sert de référence? Nous présentons brièvement ici le cas du Brésil, qui donne un aperçu de l'évolution internationale du droit, dont les enseignants doivent être conscients, quelle que soit par ailleurs I'application réelle de la loi dans les écoles publiques ou privées.

\section{Le fondement du droit: citoyenneté ou personne humaine?}

S'il est facile de défendre l'égalité comme principe de l'être humain et de la citoyenneté, il n'est pas si facile de rendre celle-ci effective puisque la diversité est visible, sensible et qu'elle semble contrevenir au principe d'égalité (" traiter tout le monde de la même façon »). Faire respecter le principe nécessite une élaboration théorique et un pacte sociopolitique transcrits dans une législation appropriée. Ainsi, la Constitution fédérale de 1988 incorpore dans son Préambule, parmi d'autres principes, celui d'assurer au Brésil une " société fraternelle et pluraliste ".

L'article 3 de la Constitution affirme que l'objectif fondamental de la République est de " promouvoir le bien de tous, sans préjugés d'origine, de race, de sexe, de couleur, d'âge et sans quelque autre forme de discrimination »; l'article 4 mentionne " le rejet du terrorisme et du racisme ". L'article 5 énumère tout ce qu'implique la défense des droits et des devoirs individuels et collectifs. Sur les 77 items qui le composent, soulignons en particulier: 
« Tous sont égaux devant la loi, sans distinction, de quelque nature qu'elle soit, garantissant aux Brésiliens et aux étrangers résidants dans le pays, l'inviolabilité du droit à la vie, à la liberté, l'égalité, à la sécurité et à la propriété [...].

I - Les hommes et les femmes sont égaux en droits et en obligations, selon les termes fixés par cette Constitution.

III - Personne ne sera soumis à la torture ni à des traitements inhumains ou dégradants.

XLI - La loi punira toute discrimination qui atteindrait les droits et libertés fondamentales.

XLII - La pratique du racisme constitue un crime non sujet à caution et imprescriptible, donnant lieu à des peines de réclusion dans les termes fixés par la loi ».

D'après cet article, les normes définies sont exécutables par le Ministère public, garant de l'ordre juridique, du régime démocratique et des droits individuels et sociaux (article 127). L'existence de tels principes n'exclut pas les autres droits et garanties qui seront signés ou ratifiés par le Brésil dans les traités internationaux.

En ce sens, notre Constitution admet comme principe supérieur la défense de la dignité de la personne, plaçant le concept même de personne humaine au-dessus du concept de citoyenneté. L'article 1 de la Constitution signale comme un des fondements de I'«État démocratique de Droit », la « dignité de la personne humaine » et le « pluralisme politique ». Les personnes sont d'emblée titulaires de droits fondamentaux, non pas parce qu'elles sont membres d'une société nationale spécifique (citoyens) mais parce que, comme le dirait Hegel: "L'homme a de la valeur parce qu'il est un homme, pas parce qu'il est juif, catholique, protestant, allemand, italien [...]».

Une fois les droits fondamentaux garantis et reconnus à toute personne humaine, quelle qu'elle soit, les droits de la citoyenneté sont confortés (article 19, III), puisqu'on affirme qu'ils sont applicables dans l'espace national, où il est interdit aux pouvoirs publics de créer des distinctions parmi les Brésiliens ou des préférences entre eux.

$D^{\prime}$ autres droits sont spécifiés dans le chapitre des Droits sociaux et énumérés dans I'article 6, dans lesquels convergent des droits de l'être humain et du citoyen. Parmi ces droits convergents qui affirment l'inclusion, on trouve l'article 205 de la Constitution sur l'éducation:

"L'éducation, droit de tous, devoir de l'État et de la famille, sera promue et encouragée avec la collaboration de la société, envisageant le plein développement de la personne, sa préparation pour l'exercice de la citoyenneté et sa qualification pour le travail. » 


\section{Droit à l'égalité et droit à la différence}

Nous avons ainsi un jeu entre " humanité et citoyenneté ». Et sous-jacent à ce jeu, il en existe un autre, en matière de conquête légale du droit, comme le souligne Norberto Bobbio, spécialiste reconnu de philosophie du droit. Dans le titre de son livre, L'ère des droits (1992), il indique ce changement par le passage du singulier au pluriel:

"Au-delà du processus de conversion en droit positif, de généralisation et d'internationalisation [...], s'est manifestée ces dernières années une nouvelle tendance, que l'on peut appeler spécification; elle consiste à déterminer de façon graduelle, mais chaque fois plus accentuée, des sujets titulaires de droits [...]. Cette spécification a eu lieu en ce qui concerne le genre, les différentes phases de la vie, ou encore la différence entre l'état normal et états exceptionnels dans l'existence humaine [...]. Par rapport aux états normaux et exceptionnels, on a fait valoir l'exigence de reconnaître des droits spéciaux aux malades, aux handicapés, aux malades mentaux etc. » (p. 62-63).

Cela veut dire qu'une fois mis en place le principe de l'égalité, il faudra se tourner vers le droit à la différence. Ainsi, la Constitution reconnaît des droits spécifiques des femmes sur le marché de travail (articles 5, item XX), interdit la différence de salaires pour des " motifs de sexe, âge, couleur ou état civil » (item XXX), la discrimination de salaire et de critères d'admission pour fait de " handicap » (item XXXI) et ce dernier item réserve un " pourcentage de postes et d'emplois publics » aux handicapés. Dans le domaine du droit à l'éducation scolaire, le travail des mineurs est interdit avant l'âge de 16 ans, pour qu'ils puissent suivre la scolarité obligatoire et l'article 208, III de la Constitution précise la nécessité d'un « accueil éducatif spécialisé pour les handicapés, de préférence dans les réseaux d'enseignement régulier ».

Les LDB de 1996 (Lois d'orientation et base de l'éducation nationale, $n^{\circ}$ 9.394/ 96) (1) réaffirment les principes de l'égalité et de la différence. La loi introduit la référence à la " tolérance " comme principe de l'éducation et, entendu comme " gestion démocratique », comme principe inhérent à l'enseignement public. L'article 4 reconnaît le besoin d'une éducation spécialisée gratuite « aux élèves à besoins particuliers, de préférence dans le réseau régulier d’enseignement ». Cette spécificité est rappelée dans les articles $37,38,58,59$ et 60 .

1 - On trouvera tous les textes (PDF) cités de la législation sur le site du ministère de l'Éducation <http//portal.mec.gov.br/index.php ?option=content\&task=view\&id=159\&ltemid=31 l> 
À son tour, le Plan national d'éducation (Loi 10.172 de 09/01/01) prévoit des entrées spéciales (diagnostics, lignes directrices, objectifs et buts) pour les modalités d'enseignement concernant l'éducation de jeunes adultes, l'éducation à distance, les technologies éducatives, la formation professionnelle, l'éducation spéciale et l'éducation indigène.

\section{Les droits des enfants handicapés aux réseaux d'enseignement}

Dans le cas de l'éducation spéciale, l'organisation juridique a avancé du fait que le Congrès national a ratifié en 2001 le texte de la Convention interaméricaine pour I'élimination de toutes les formes de discrimination à l'encontre des personnes ayant un handicap (Décret du 13 juin 2001). Promulgué par le président de la République, le décret du 8 octobre 2001 vise à prévenir et éliminer la discrimination « sous toutes ses formes et manifestations, à l'encontre des personnes handicapées et à favoriser leur pleine intégration dans la société ».

Cependant, contrairement aux pays d'Europe, le Brésil ne disposait pas d'un réseau public développé pour l'éducation spécialisée. Pour la France, par exemple, la loi tend à transférer des enfants auparavant orientés vers des institutions et classes " spéciales » vers des classes ordinaires où ils doivent désormais " être inclus » dans leur classe d'âge. Au Brésil, elle rend légale la scolarisation d'un nouveau public d'enfants, jusque-là scolarisés de façon aléatoire, selon la plus ou moins grande volonté des familles, leurs ressources financières et les offres de l'environnement (réseaux d'institutions privées).

La Direction de l'éducation de base a donc dû interpréter la loi de 1996 pour rendre applicables les articles concernant l'éducation spéciale. Elle a rédigé en 2001 une circulaire et une résolution qui constituent les « Directives nationales de la grille scolaire de l'éducation spéciale » en tant que modalité de l'éducation de base. De ce fait, les directives concernant l'éducation des élèves à besoins éducatifs particuliers ne sont pas adressées à une (inexistante) direction chargée de l'enseignement spécial. Les directives de cette Circulaire ne s'ajoutent pas aux Directives de l'éducation de base, elles s'appliquent sans distinction à tous les élèves en cours de scolarisation, parmi lesquels ceux qui ont des besoins particuliers. Elles insistent sur la nécessité de promouvoir une éducation pour l'égalité dans la diversité et font de l'inclusion démocratique une forme de lutte contre l'inégalité et contre toutes les formes de discrimination contre la personne humaine. 
Cette prise de position axiologique s'appuie sur la juridiction aujourd'hui la plus avancée contre toute discrimination, tout en préservant la complexité du réel qui ne relève jamais d'un jugement en " tout ou rien ».

« Les perspectives énoncées dans la Circulaire du CNE (2)/CEB n 17/2001, permettent de préserver la diversité dans l'égalité et doivent être un guide pour les dispositifs. Les différences, et dans le cas qui nous concerne, celles qui affectent les élèves à besoins éducatifs particuliers, sont compatibles avec la clause d'égalité non pas parce que cela est accepté explicitement par la loi fondamentale, mais parce que la loi prend en considération le lien logique existant entre les différences et l'égalité qu'elle se doit justement de préserver. Ces diversités montrent qu'il est nécessaire de créer une nouvelle culture: l'éducation inclusive, qui, en identifiant des discriminations ségrégatives de toutes sortes, vise à empêcher leur perpétuation et en même temps, à établir une nouvelle conception de l'égalité, en introduisant une obligation clairement exprimée à l'art. 7 de la Résolution CNE/CEB 02/2001 : "L'accueil des élèves à besoins éducatifs particuliers doit (3) être réalisé dans les classes ordinaires de l'enseignement régulier commun, quels que soient les niveaux ou les modalités de l'éducation de base. "

De même, l'article 2 de la même résolution stipule:

«Les systèmes d'enseignement doivent inscrire tous les élèves, et il revient aux écoles de s'organiser pour accueillir les élèves ayant des besoins particuliers, en assurant les conditions nécessaires pour une éducation de qualité pour tous. »

46 Si la Constitution plaide pour un accueil éducatif dans le réseau régulier de l'enseignement, il faut faire attention à la portée de cette formulation. Accueillir, c'est être disponible pour recevoir ou, dans notre perspective d'inclusion, pour ne pas laisser dehors. Mais il s'agit d'un accueil dans l'éducation scolaire lqui comporte plusieurs réseaux d'enseignement) et dans le réseau "régulier » d'enseignement. "Régulier 》 s'oppose à « irrégulier» (hors des dispositifs légaux) et aussi à « libre » (qui ne se trouve pas régi par la loi scolaire mais prétend, au sens large du terme, offrir une éducation). En même temps, rien n'empêche qu'il y ait d'autres modalités d'enseignement et d'éducation conçues pour préserver ou prendre en compte une différence qui mérite une approche spécifique du fait de ses caractéristiques propres.

2 - CNE: Conselho nacional de educação = Conseil national de l'éducation/CEB : Câmara de Educação Básica = Chambre/Bureau de l'éducation fondamentale.

3 - Souligné par nous. 
La recommandation demande donc de considérer " l'homme aussi bien en tant qu'être générique à qui le principe de l'égalité s'applique sans discriminations, qu'en tant qu'être humain dans une situation concrète au moment de l'application de la norme universelle.

Par conséquent, les États de droit démocratique signalent quelles discriminations doivent toujours être interdites: celles qui concernent l'origine, la race, le sexe, la religion, la couleur, les croyances. Mais en même temps, il serait absurde de viser l'égalitarisme, une égalité absolue, qui imposerait $d^{\prime}$ 'appliquer les lois de manière uniforme à tous les sujets et dans toutes les situations.

Un traitement différencié ne se justifie que devant une situation objective et rationnelle, en prenant en compte le contexte large de son application. La différence de traitement doit être en rapport avec la situation et avec la finalité de la loi et elle doit être assez claire et logique pour se justifier ».

La valeur des articles de loi conserve en permanence son horizon égalitaire, mais un accueil différencié des personnes handicapées doit, dans certaines circonstances, être préservé. Et cette protection concerne le moment d'application de la loi, nommé «équité » (4).

\section{De l'égalité des principes à la recherche de l'équité}

Le concept $d$ '«équité » cherche à maintenir l'équilibre entre le principe de l'égalité et les conditions concrètes de son application en tenant compte de la diversité des situations et des individus. L'équité constitue la médiation par rapport à la norme égalitaire quand son application pourrait contrevenir à l'égalité elle-même ou lorsque la situation est un " cas particulier». Dans le cas concret où se révèle une situation potentiellement source d'obstacles à l'égalité, on doit chercher son dépassement, de manière à introduire un rapport plus égalitaire (ou moins inégalitaire), là où il y avait auparavant rapport d'inégalité (5).

Comme l'écrit Aristote, «L'équité est juste, toutefois pas légalement juste, mais apportant une correction de

4 - Cf. Cury, 1999.

5 - On peut rappeler la maxime ancienne "Summum Jus, Summum injuria » («l'excès de justice produit l'injustice») citée par Cicéron (De Officiis, I, 10,33) pour dénoncer les injustices découlant d'une application aveugle et uniforme des lois, qui a comme conception préalable une vision égalitariste de tous les individus. 
la justice légale [...], c'est cela, la nature de l'équité: une correction de la loi quand elle est déficiente en raison de son universalité $»$.

L'équité consiste donc à rechercher une adéquation entre la loi et sa mise en contexte pour résoudre " au cas par cas », avec prudence, des cas non réglés par le caractère trop large de la loi universelle. Pour Aristote, c'est la nature complexe de l'objet ou de la situation, confrontée au caractère absolu de la loi qui, face au besoin d'être juste, postule le principe de l'équité. Pour lui, dans certaines circonstances, l'équité traduit mieux l'exigence de justice que l'égalité prise en tant qu'égalitarisme. L'équité est un antidote à l'homogénéisation normalisatrice et à la crispation identitaire. Dans le cas de l'éducation inclusive, cela nous conduit à faire une distinction importante entre le moment juridique et le moment pédagogique. Les deux concourent à la même fin. Le moment juridique dit la norme, affirme le principe et se déploie pour permettre au moment pédagogique de le rendre effectif. Celui-ci, à son tour, comprend la préparation appropriée de l'environnement scolaire et du corps enseignant, de manière à ne pas ajourner les mesures nécessaires pour traiter à la fois l'égalité et les besoins particuliers, le rejet de toute discrimination et la prise en compte des différences. Le moment pédagogique ne peut empêcher, sous aucun prétexte, l'accueil dans les écoles des enfants à besoins particuliers.

\section{Reconnaître les différences, rejeter les discriminations}

De même, en identifiant historiquement diverses cultures brésiliennes dans notre formation socioculturelle, la Constitution n'a pas seulement eu l'intention de les préserver de l'oubli mais a voulu les reconnaître comme une richesse qu'il faut valoriser. D'où la condamnation du racisme et des préjugés raciaux existant au Brésil.

Le rapport entre la condamnation des pratiques discriminatoires et l'affirmation des droits a été mis en évidence par Bobbio (1987). Selon lui, la pluralité valorisée de manière affirmative gagne en substance chaque fois qu'elle sert à mettre à bas une discrimination basée sur une modalité de préjugé quelconque. C'est dans ce sens qu'il indique une dialectique entre liberté et égalité:

" Je considère la liberté socialiste par excellence comme celle qui, en libérant, égalise, et elle égalise quand elle élimine une discrimination; c'est une liberté qui n'est pas seulement compatible avec l'égalité, mais qui en est la condition. » (p. 23)

Toutes les formes qui conçoivent l'égalité comme uniformité ignorent la valeur des différences ou les restreignent à l'espace privé, et évoluent vers des régimes autoritaires, dictatoriaux ou même totalitaires. Inversement, l'excessive considération des différences peut aboutir à l'effet contraire à celui qui était recherché et produire un 
appauvrissement de l'être social de l'homme. C'est ce que l'on peut vérifier dans des sociétés saisies par des fondamentalismes ou des crispations identitaires de toutes sortes, dans lesquelles, comme le dit Rouanet (1994), domine l'ontologie de la différence. En s'opposant à ces positions dogmatiques cet auteur défend ce qu'il appelle " universalisme concret».

"L'utopie des Lumières, c'est celle d'une éthique fondée sur la raison, tournée vers le bonheur, capable de juger et critiquer l'existant, et qui vise une communauté de discussion sans frontières, où l'égalité $n^{\prime}$ est pas conçue comme un nivellement et où l'universalité ne conduit pas à la dissolution du privé. » (p. 162)

La démocratie suppose aussi bien l'égalité pour ce qui est égal ou censé être égal, que la considération positive de la différence comme révélatrice de la profonde richesse dont sont revêtus tous les êtres humains, à la condition qu'une telle différence prenne place dans la matrice égalitaire de l'être humain.

L'être humain se caractérise aussi bien par sa relativité que par sa perfectibilité. Dans ce sens, nous sommes tous contingents et, par nature, nous ne nous suffisons pas. Nous sommes tous des personnes avec des besoins, qui sont autant de nécessités vitales, manifestes ou non, particulières ou non.

Aristote a donc défini le besoin comme quelque chose " par laquelle nous sommes forcés » et qui nous est nécessaire.

« Il y a nécessité quand quelque force nous oblige à faire ou subir quelque chose qui va contre notre instinct, de sorte que la nécessité consiste en ce cas à ne pas pouvoir faire autrement ou à subir autrement. » (Aristote, Métaphysique, V, 5, 1014 b 35)

Notre insertion sociale nous lie à l'autre comme un moyen qui nous permet d'assurer les conditions de base de notre existence et même de dépasser nos limitations individuelles. Personne ne peut assurer la vie à soi-même et nous ne pouvons pas dépendre isolément de notre volonté. La limitation de notre durée dans l'espace et dans le temps est la preuve majeure que nous sommes tous limités. Et c'est aussi l'appel pour notre sociabilité et la quête de l'autre.

Nous sommes tous des êtres humains singuliers. C'est ce qui nous distingue les uns des autres. Les récentes découvertes de la science nous montrent que cela a d'ailleurs un fondement biologique. Plusieurs de nos différences individuelles se manifestent directement et visiblement (l'iris des yeux, la couleur de la peau, notre stature, notre poids), d'autres non. Il y a des personnes qui présentent des handicaps, sur le plan physique ou psychique, de manière temporaire ou permanente, de manière partielle ou totale dont la réalité est visible, en particulier celles qui atteignent la dynamique sensorielle. D'après la Convention du Guatemala (2001), dans I'article I: 
"Le terme "déficience" désigne une restriction physique, mentale ou sensorielle, de nature permanente ou transitoire, qui limite la capacité d'exercer une ou plusieurs activités essentielles de la vie quotidienne, causée ou aggravée par l'environnement économique et social. »

\section{Une nouvelle culture dans la formation des maîtres et la législation scolaire}

Les élèves à besoins particuliers qui entrent dans les écoles comme les élèves ordinaires, demandent une attention spécifique, puisque leurs besoins peuvent affecter leurs modes d'apprentissage. Des processus pédagogiques appropriés peuvent réduire, voire éliminer de telles limitations, en s'appuyant sur les capacités disponibles de l'enfant et en stimulant sa motivation à apprendre.

Comme le signalent la circulaire et la résolution du Conseil national, l'éducation inclusive, en touchant aux élèves " à besoins éducatifs particuliers ", fait nécessairement entrer dans une nouvelle culture. Et c'est là que la formation des professeurs doit jouer son rôle, qu'elle soit générale et spécialisée, qu'il s'agisse de formation initiale ou de formation en cours d'emploi. C'est pourquoi chaque fois que la législation et la réglementation traitent de la formation des professeurs, l'éducation spéciale est immédiatement citée comme une obligation des institutions de formation. C'est dire que les étudiants des cours normaux, secondaires ou supérieurs, des cours de pédagogie et de licence doivent recevoir un enseignement sur le droit à la différence, dans la perspective de l'éducation spéciale et de l'éducation inclusive. Au-delà des références explicites de la législation déjà mentionnées, il y a toute la réglementation du Conseil national d'éducation.

Ainsi, l'article 59 de la LDB prévoit la formation des personnes ayant à enseigner à des publics avec des besoins éducatifs particuliers. Ce seront des " professeurs spécialisés, de niveau secondaire ou supérieur, pour l'accueil spécialisé, ainsi que des professeurs de l'enseignement régulier, habilités pour l'intégration de ces élèves dans les classes ordinaires. »

En fonction de quoi, la Résolution de février 2001 incite les établissements supérieurs de formation de professeurs à inclure des « contenus appropriés sur l'éducation spéciale qui les conduisent à maîtriser les procédures didactiques pédagogiques et les pratiques alternatives » pour accueillir ceux qui ont de tels besoins.

Cette exigence découle des "Directives nationales du cursus pour la formation de professeurs de l'éducation enfantine » et des " années initiales de l'enseignement fondamental » (Résolution CEB/CNE 02/99) et des Directives nationales du cursus pour la formation de professeurs pour l'éducation de base en niveau supérieur 
(Résolution CP/CNE 01/02). Elles stipulent que dans la composition du cursus et dans l'acquisition et la maîtrise des savoirs, valeurs, capacités et compétences dans cette formation, il est indispensable d'accueillir la diversité, en particulier celle des individus qui ont des « besoins particuliers ».

Le chapitre consacré à l'éducation spéciale dans le Plan national d'éducation (Loi de 2001) a réitéré cette exigence, s'agissant du diagnostic, des directives, des objectifs et a défini 28 cibles à atteindre dans des délais fixés. Ainsi, la cible $n^{\circ} 19$ exige dans les cours de formation des professeurs, l'inclusion de contenus et de disciplines spécifiques pour l'habilitation à la réception des élèves spéciaux. La cible $\mathrm{n}^{\circ} 20$ oblige les universités publiques à créer une habilitation spécifique pour la formation du personnel spécialisé qui recevra les élèves à besoins particuliers.

D'autres composantes juridiques ont complété ce cadre, comme la Loi de 2002, sur la langue des signes (LIBRAS), obligeant les systèmes d'enseignement à rendre obligatoire l'enseignement de cette langue des signes dans la formation des professeurs et à créer un cours supérieur pour la formation de professeurs ayant cette compétence.

Une loi de 2007 a prévu que dans le fonds de l'éducation de base un pourcentage du budget soit affecté à l'éducation spéciale.

Comme on peut le remarquer, si l'éducation spéciale reste en souffrance, ce ne sera pas par manque de lois et de décrets! Bien au contraire, le dispositif juridique donne une importante couverture réglementaire à cette modalité d'éducation scolaire, autant qu'à la formation des professeurs concernés.

\section{Conclusion}

Former des professeurs ne résoudra pas tous les problèmes. II faut lutter contre le manque d'information des familles, faire passer le droit dans les faits et faire en sorte d'atteindre les cibles du Plan national d'éducation, et il y a encore beaucoup à faire dans le domaine de la formation. Pourtant, la formation des professeurs ne peut ni esquiver les urgences propres à la société actuelle, avec son formidable environnement technologique, ni son engagement dans la " mise à niveau 》 des valeurs citoyennes et la participation de l'éducation scolaire à ces nouveaux défis.

La responsabilité des institutions de formation est donc en première ligne pour ce qui est de faire naître cette nouvelle culture de la différence, de manière à déconstruire des préjugés, à construire la dynamique du respect (qui inclut justement le droit à une formation professionnelle qualifiée). Mais il est aussi important de souligner que dans les différents États du Brésil, les gouvernements sont encore très éloignés des politiques et des actions programmatiques qui puissent faire passer ce droit dans la réalité. 
La dialectique entre le droit à l'égalité et le droit à la différence dans l'éducation scolaire en tant que devoir de l'État et droit du citoyen n'est plus un rapport simple. D'un côté, il faut faire la défense de l'égalité en tant que principe de citoyenneté, de la modernité et des droits humains. L'égalité est aussi bien le principe de la non-discrimination, que le principe central pour lequel les hommes ont lutté pour éliminer les privilèges de sang, d'ethnie, de religion ou de croyance. Elle est encore ce qui oriente les personnes en lutte pour réduire les inégalités et éliminer peu à peu les différences discriminatoires (6).

L'accès universel à l'éducation scolaire est une forme de socialisation institutionnelle, un facteur de convivialité permettant aux enfants, aux adolescents et à tous les jeunes et adultes, l'expression d'émotions et la construction de savoirs, de valeurs et de compétences qui aident à dépasser l'égocentrisme, à promouvoir le respect d'autrui et la réciprocité. La citoyenneté ne devient "adulte » que quand la personne se confronte à des situations où le respect de ses propres droits se trouve limité par le respect dû aux droits d'autrui.

Dans le fond, ce que cette loi oblige à considérer concrètement, c'est l'altérité et l'existence de l'(des) autre(s) en tant qu'égaux. L'éducation inclusive, dans cette mesure, est une belle occasion de croissance de la citoyenneté et une clef pour une meilleure estime de soi.

6 - Cf. Cury, 2002. 


\section{BIBLIOGRAPHIE}

ARISTOTELES (1973). Ética a Nicômaco, V, 10, 1137 b., in Coleção Os Pensadores, São Paulo: Abril Cultural.

ARISTOTELES (1952). "Metaphysics", V, 5, 1014 b 35, in The Great Books, Oxford: Encyplopaedia Britannica, Inc.

BOBBIO Norberto (1987). "Reformismo, socialismo e igualdade", Novos Estudos, n 19, São Paulo: CEBRAP.

BOBBIO Norberto (1992). A Era dos direitos, Rio de Janeiro: Campus.

CURY Carlos Roberto Jamil (1999). "Direito à diferença: um reconhecimento legal", Educação em Revista, $n^{\circ}$ 30, Belo Horizonte, p. 7-15.

CURY Carlos Roberto Jamil (2002). "Direito à educação: direito à igualdade, direito à diferença", Cadernos de Pesquisa, São Paulo, n 116, p. 245-262.

ROUANET Sergio Paulo. (1994). "Dilemas da Moral Iluminista", in Novaes Adauto [dir.] Ética, São Paulo: Cia das Letras. 
